\title{
The "Traditional Chinese medicine regulating liver regeneration" treatment plan for reducing mortality of patients with hepatitis B-related liver failure based on real-world clinical data
}

\author{
Ling Dai ${ }^{*}$ Xiang Gao ${ }^{*}$ Zhihua Ye*, Hanmin Li (凶), Xin Yao, Dingbo Lu, Na Wu \\ Institute of Liver Diseases, Hubei Provincial Hospital of Traditional Chinese Medicine, Wuhan 430061, China; Institute of Basic Theory of \\ Chinese Medicine, Hubei Province Academy of Traditional Chinese Medicine, Wuhan 430074, China; Theory and Application Research of \\ Liver and Kidney in Traditional Chinese Medicine, Hubei Provincial Key Laboratory, Wuhan 430074, China; Key Laboratory of Treating \\ Chronic Liver Diseases from Liver and Kidney, State Administration of Traditional Chinese Medicine, Wuhan 430061, China
}

(C) Higher Education Press 2020

\begin{abstract}
On the basis of real-world clinical data, the study aimed to explore the effect and mechanisms of the treatment plan of "traditional Chinese medicine (TCM) regulating liver regeneration." A total of 457 patients with HBV-related liver failure were retrospectively collected. The patients were divided into three groups: the modern medicine control group (MMC group), patients treated with routine medical treatment; the control group combining traditional Chinese and Western medicine (CTW), patients treated with routine medical treatment plus the common TCM formula; and the treatment group of "TCM regulating liver regeneration" (RLR), patients treated with both routine medical treatment and the special TCM formula of RLR. After 8 weeks of treatment, the mortality of patients in the RLR group (12.31\%) was significantly lower than those in the MMC (50\%) and CTW (29.11\%) groups. Total bilirubin level significantly decreased and albumin increased in the RLR group when compared with the MMC and CTW groups $(P<0.05)$. In addition, there were significant differences in the expression of several cytokines related to liver regeneration in the RLR group compared with the MMC group. RLR treatment can decrease jaundice, improve liver function, and significantly reduce the mortality in patients with HBV-related liver failure. The mechanism may be related to the role of RLR treatment in influencing cytokines related to liver regeneration.
\end{abstract}

Keywords hepatitis B virus-related liver failure; traditional Chinese medicine; liver regeneration; liver regeneration microenvironment; cytokines

\section{Introduction}

Liver failure is a common critical disease of the digestive system. The typical clinical manifestations include coagulopathy, jaundice, hepatic encephalopathy, and ascites. However, there are no effective drugs to treat patients with liver failure. Patients in the late stage of liver failure often suffer from various complications, including electrolyte disorders, refractory ascites, and hepatic encephalopathy, all of which contribute to high mortality [1]. Drug-

Received June 21, 2019; accepted April 17, 2020

Correspondence: Hanmin Li, lihanmin69@126.com

"Ling Dai, Xiang Gao, and Zhihua Ye contributed equally to this work. mediated hepatic toxicity or excessive alcohol consumption is the main cause of liver failure in European and American countries, while more than $80 \%$ of patients with liver failure in China are related to hepatitis B virus (HBV) infection [2,3]. Patients with HBV-related liver failure are often in severe conditions and accompanied with various complications. The pathogenesis of HBV-related liver failure, which involves the interaction of multiple factors, has not been fully elucidated. The interactions of the virus and host factors are considered the main pathogenic reason for the disease. These viral factors include genotype, replication efficiency, and mutations. The host factors include, but are not limited to, genetic background, the mechanisms of pathological damage protection, and abnormal liver regeneration [4,5]. Previous studies have found that the treatment of "traditional Chinese medicine 
(TCM) regulating liver regeneration" (abbreviated as RLR) promotes liver regeneration and repairs injured hepatic parenchyma by affecting stem cells. Thus, the effectiveness of liver regeneration and repair has become the key determinant for the survival of patients with liver failure [6].

The present study aimed to investigate the efficacy and mechanism of the RLR add-on in the treatment of HBVrelated liver failure by retrospectively analyzing clinical data. In addition, the serum hemopoietic growth factors (HGFs) were monitored using suspension array technology. The present results suggested that the treatment of "TCM regulating liver regeneration" can significantly reduce the mortality of patients with HBV-related liver failure.

On the basis of the clinical data of real-world research, this study aimed to investigate the efficacy of the RLR program in the treatment of HBV-related liver failure. In addition, the serum cytokine levels associated with liver regeneration were monitored by suspension array technology to explore the therapeutic mechanism of the RLR program in reducing the mortality of patients with HBVrelated liver failure by improving the microenvironment of liver regeneration.

\section{Material and methods}

\section{Patients}

This work is a retrospective study based on real-world clinical data. A total of 457 medical records of patients with HBV-related liver failure from July 2012 to May 2018 were collected by retrieving the electronic medical record system of the Hubei Provincial Hospital of Traditional Chinese Medicine. Patients with acute liver failure or subacute liver failure have acute onset, rapid progression, and high mortality in the short term. They are not suitable for clinical observation of drugs, so this study did not include patients with acute liver failure. Patients with liver failure in this study were specifically referred to patients with acute (subacute)-on-chronic liver failure (abbreviated as ACLF or SACLF) or chronic liver failure (CLF). According to the clinical treatment plan, all included patients were divided into three groups: the modern medicine control group (MMC group), the control group combining traditional Chinese and Western medicine (CTW group), and the group of "TCM regulating liver regeneration" (RLR group). The study flow diagram is shown in Fig. 1.

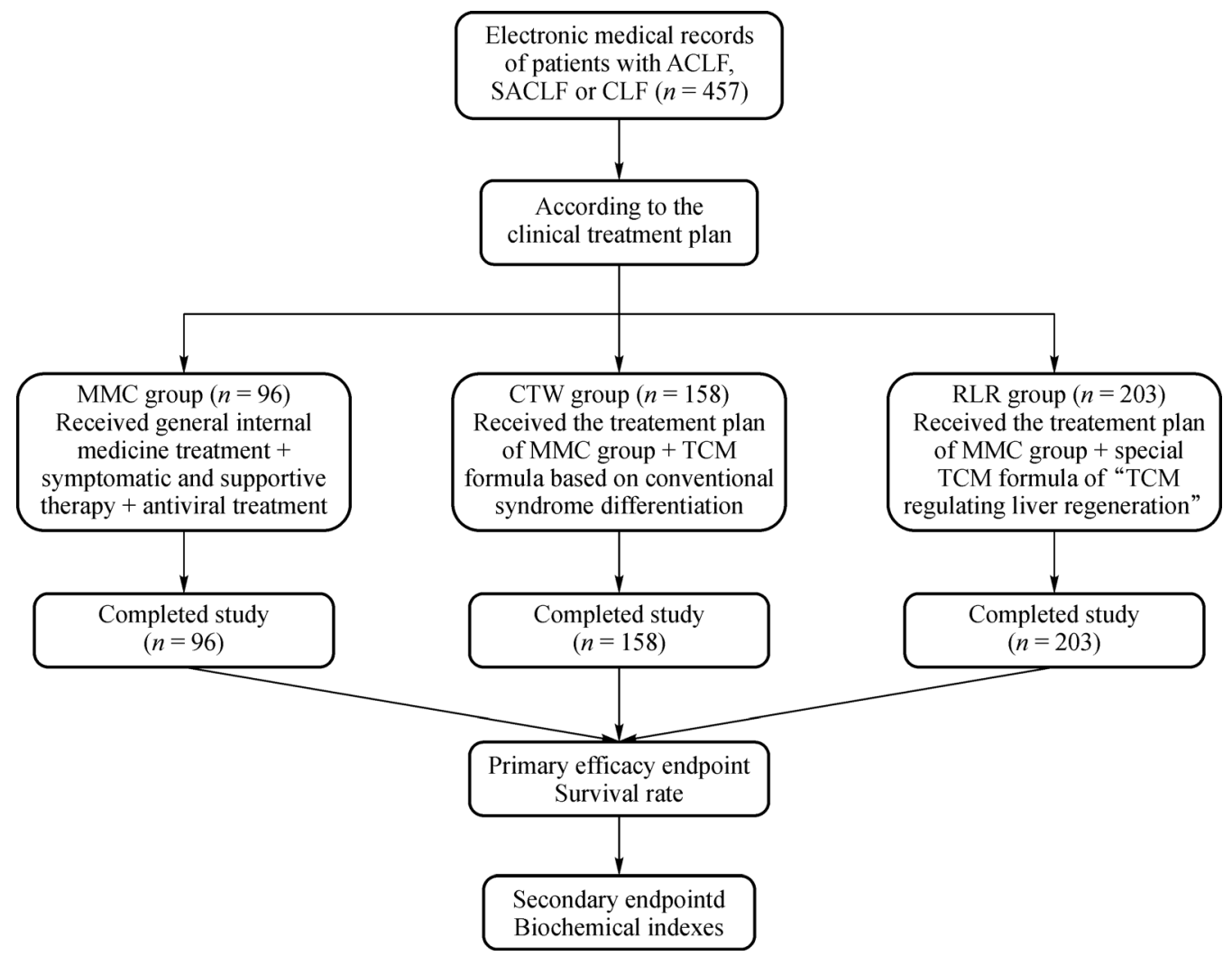

Fig. 1 Trial flow diagram. ACLF, acute-on-chronic liver failure; SACLF, subacute-on-chronic liver failure; and CLF, chronic liver failure. 


\section{Inclusion criteria}

Patients with HBV-related ACLF (SACLF) or CLF were included in this study. Liver failure was diagnosed based on the diagnostic criteria established in the 2012 version of the Diagnosis and Treatment Guidelines for Liver Failure [7]. Patients who fulfilled the following criteria were diagnosed with ACLF/SACLF: (1) patients were extremely weak, with obvious symptoms of digestive tract; (2) patients exhibited a rapid increase of jaundice, and serum TBIL levels were greater than 10 times the upper limit of normal or daily increase $\geqslant 17.1 \mu \mathrm{mol} / \mathrm{L}$; (3) patients presented with bleeding tendency (PTA $\leqslant$ $40 \%$ or INR $\geqslant 1.5$ ), and other causes were excluded; (4) patients with decompensated ascites; and (5) patients with/without hepatic encephalopathy. Patients who fulfilled the following criteria were diagnosed with CLF: (1) patients with significantly increased serum TBIL levels; (2) patients with significantly reduced albumin levels; (3) patients with bleeding tendency (PTA $\leqslant 40 \%$ or INR $\geqslant 1.5)$, and other causes were excluded; (4) patients with ascites or portal hypertension; and (5) patients with hepatic encephalopathy. Exclusion criteria were as follows: (1) patients with acute liver failure; (2) patients with primary hepatic carcinoma; (3) patients with other hepatotropic virus infections, including hepatitis A virus, hepatitis $C$ virus, hepatitis $D$ virus, hepatitis $E$ virus, Epstein-Barr virus, and cytomegalovirus infection; (4) patients with liver failure caused by diseases other than chronic hepatitis B; and (5) patients who were lactating or pregnant.

\section{Therapeutic plans}

The treatment plan of the MMC group consisted of general basic treatment, symptomatic and supportive therapy, and antiviral treatment. The general basic treatment included the compound glycyrrhizin for injection (Harbin Sanlian Pharmaceutical Co., Ltd., 80-160 mg, once a day, intravenous drip), reduced glutathione for injection (Shanghai Fudan Fuhua Pharmaceutical Co., Ltd., Medicine Co., Ltd., $1.2 \mathrm{~g}$, once a day, intravenous drip) or acetylcysteine injection (Ruiyang Pharmaceutical Co., Ltd., $4.0 \mathrm{~g}$, once a day, intravenous drip), and hepatocyte growth-promoting factor for injection (Guangzhou Yipinhong Pharmaceutical Co., Ltd., 80-120 mg, once a day, intravenous drip). Symptomatic and supportive therapy can actively prevent and mitigate hepatic encephalopathy, cerebral edema, hepatorenal syndrome, infections, gastrointestinal bleeding, and other complications. The antiviral treatment includes the oral administration of nucleoside antiviral drugs of lamivudine (100 mg, once a day) or entecavir (Zhengda Tianqing Pharmaceutical Group Co., Ltd., $0.5 \mathrm{mg}$, once a day). Adefovir dipivoxil $(10 \mathrm{mg}$, once a day) or entecavir (1 mg, once a day) was prescribed to patients who developed lamivudine resistance.

The treatment plan of the CTW group combined the treatment plan of the MMC group with the TCM formula based on conventional syndrome differentiation. The basic formula in the CTW group consisted of the following: $30 \mathrm{~g}$ of processed Astragalus root, 30-60 g of Polygonum cuspidatum, $30 \mathrm{~g}$ of Indian buead, $30 \mathrm{~g}$ of Salvia miltiorrhiza root, $30 \mathrm{~g}$ of motherwort herb, $20 \mathrm{~g}$ of Grifola, $30 \mathrm{~g}$ of parched white Atractylodes rhizome, $30-60 \mathrm{~g}$ of virgate wormwood herb, $12 \mathrm{~g}$ of Cape jasmine fruit, $6 \mathrm{~g}$ of Baikal skullcap, $10 \mathrm{~g}$ of rhubarb, and $6 \mathrm{~g}$ of licorice root.

The treatment plan of the RLR group combined the MMC treatment plan with the special TCM formula of "TCM regulating liver regeneration." The basic formula of the RLR group included 15-30 g of prepared Rehmannia root, 30-60 $\mathrm{g}$ of virgate wormwood herb, 3-6 $\mathrm{g}$ of turmeric, $10-15 \mathrm{~g}$ of Chinese Magnoliavine fruit, $9-12 \mathrm{~g}$ of licorice root, $15 \mathrm{~g}$ of common yam rhizome, $15 \mathrm{~g}$ of barbary wolfberry fruit, $15 \mathrm{~g}$ of common macrocarpium fruit, $10 \mathrm{~g}$ of south dodder seeds, $30 \mathrm{~g}$ of Indian buead, $10 \mathrm{~g}$ of tree peony bark, and $10 \mathrm{~g}$ of oriental water plantain rhizome. The regular composition of the TCM formula was subjected to addition or subtraction on the basis of the differentiation of the main symptoms.

All components of TCM formulas were stewed in water, resulting in a $260 \mathrm{~mL}$ lukewarm decoction, and $130 \mathrm{~mL}$ per drink was given twice a day. The same formulation was renewed daily.

\section{Endpoints}

The primary endpoint of clinical efficacy was the survival rate. The secondary endpoint was the improvement of biochemical indexes (alanine aminotransferase (ALT), total bilirubin (TBIL), albumin (ALB), and prothrombin activity (PTA)) that were tested by using a German AMAX-200 automatic coagulation analyzer with serum quality control and the supplied reagent detection accessories and a Toshiba 120 automatic biochemical analyzer with the supplied reagents and detection accessories. The detection and quality control of biochemical indicators were completed by the Laboratory Department of Hubei Provincial Hospital of Traditional Chinese Medicine.

\section{Cytokine detection}

The serum cytokines were detected using the Bio-plex suspension array system. The PDGFbb, IL-2, IL-6, IL-10, IL-13, FGF-basic, GCSF, GMCSF, interferon (IFN)- $\gamma$, TNF- $\alpha$, IL-18, LIF, MIF, $\beta$-NGF, stem cell factor (SCF), TRAIL, HGF, TGF- $\beta 1$, vascular endothelial growth factor (VEGF), and IL-12 kits and Bio-plex suspension array system were provided by Bio-Rad Laboratories (USA). 
The serum samples in the present study were obtained from normal controls (NCs), who were healthy individuals that underwent annual checkups at the Physical Examination Center of Hubei Provincial Hospital of Traditional Chinese Medicine. All serum samples were stored at $-80{ }^{\circ} \mathrm{C}$ until analysis.

\section{Statistical methods}

Statistical analysis was performed using SPSS 19.0 software. Mean \pm standard deviation $(\bar{x} \pm s)$ was used to describe the quantitative data, while one-way ANOVA was used to determine the significant differences. $X^{2}$-test was used for categorical data. $P<0.05$ was considered statistically significant.

\section{Results}

\section{Baseline of patients}

All medical records were electronically retrieved and collected from the medical record system of Hubei Provincial Hospital of Traditional Chinese Medicine. The demographics and clinical diagnoses of patients in these three groups at baseline are listed in Table 1.

\section{Antiviral drugs and percentages of patients receiving antiviral therapy}

The antiviral drugs and percentages of patients receiving antiviral therapy in each group $(n(\%))$ are listed in Table 2.
There was no significant difference in the percentage of patients receiving antiviral treatment among the three groups $(P>0.05)$.

\section{Reduction in mortality in groups treated with CTW and RLR}

After the 8-week treatment period, the mortality rate was computed in each group, and the following values were attained: $50 \%$ in the MMC group, $29.11 \%$ in the CTW group, and $12.31 \%$ in the RLR group (Fig. 2). The differences in mortality among these three groups were significant $(50 \%$ vs. $29.11 \%$ vs. $12.31 \% ; P<0.05)$, implying that mortality in the RLR group was significantly reduced when compared with both the CTW and MMC groups $(12.31 \%$ vs. $29.11 \%$ and $12.31 \%$ vs. $50 \% ; P<$ $0.01)$. Notably, the mortality of the RLR group was lower than that of the two other groups in both ACLF (SACLF) and CLF, and the difference was statistically significant $(P<0.01)$.

\section{Improved biochemical indexes in groups treated with RLR}

After the 8-week treatment period, the serum ALB level was significantly higher in the RLR group than in the two other groups $(35.09 \pm 6.42$ vs. $32.44 \pm 5.90$ and $33.11 \pm 5.43 ; P<0.05)$. Furthermore, serum TBIL was significantly lower in the RLR group than in the MMC and CTW groups $(155.42 \pm 176.17$ vs. $224.96 \pm 198.61$ and $233.25 \pm 226.16 ; P<0.05)$. In addition, the serum ALT levels in all three groups significantly decreased when

Table 1 Demographic and clinical diagnosis of the three groups

\begin{tabular}{|c|c|c|c|c|}
\hline Characteristic & $\begin{array}{l}\mathrm{MMC} \\
(n=96)\end{array}$ & $\begin{array}{l}\text { CTW } \\
(n=158)\end{array}$ & $\begin{array}{l}\text { RLR } \\
(n=203)\end{array}$ & $P$ value \\
\hline$\overline{\text { Age (year, } \bar{x} \pm s \text { ) }}$ & $47.65 \pm 12.96$ & $47.68 \pm 12.91$ & $47.32 \pm 12.98$ & 0.959 \\
\hline Male $(n(\%))$ & $79(82.29)$ & $128(81.01)$ & $168(82.76)$ & 0.91 \\
\hline Disease course (year, $\bar{x} \pm s$ ) & $11.84 \pm 11.98$ & $11.16 \pm 10.52$ & $12.24 \pm 9.76$ & 0.63 \\
\hline Acute-on-chronic (subacute) liver failure $(n(\%))$ & $55(57.29)$ & $84(53.16)$ & $103(50.74)$ & 0.569 \\
\hline Chronic liver failure $(n(\%))$ & $41(42.71)$ & $74(46.84)$ & $100(49.26)$ & 0.569 \\
\hline
\end{tabular}

Table 2 Antiviral drugs and percentages of patients receiving antiviral therapy in each group (n (\%))

\begin{tabular}{llll}
\hline Antiviral drug & $\begin{array}{l}\text { MMC } \\
(n=96)\end{array}$ & $\begin{array}{l}\text { CTW } \\
(n=158)\end{array}$ & $\begin{array}{l}\text { RLR } \\
(n=203)\end{array}$ \\
\hline No antiviral drugs used & $30(31.25)$ & $45(28.48)$ & $64(31.53)$ \\
Lamivudine & $23(23.96)$ & $40(25.32)$ & $32(15.76)$ \\
Adefovir dipivoxil & $0(0.00)$ & $11(6.96)$ & $17(8.37)$ \\
Telbivudine & $4(4.16)$ & $12(7.59)$ & $8(3.94)$ \\
Entecavir & $34(35.42)$ & $43(27.22)$ & $69(33.99)$ \\
Lamivudine plus adefovir dipivoxil & $3(3.13)$ & $4(2.53)$ & $7(3.45)$ \\
Entecavir plus adefovir dipivoxil & $2(2.08)$ & $3(1.90)$ & $6(2.96)$ \\
Antiviral drugs used (total) & $66(68.75)$ & $113(71.52)$ & $139(68.47)$ \\
\hline
\end{tabular}

There was no significant difference in the percentage of patients receiving antiviral treatment among the three groups $(P>0.05)$. 

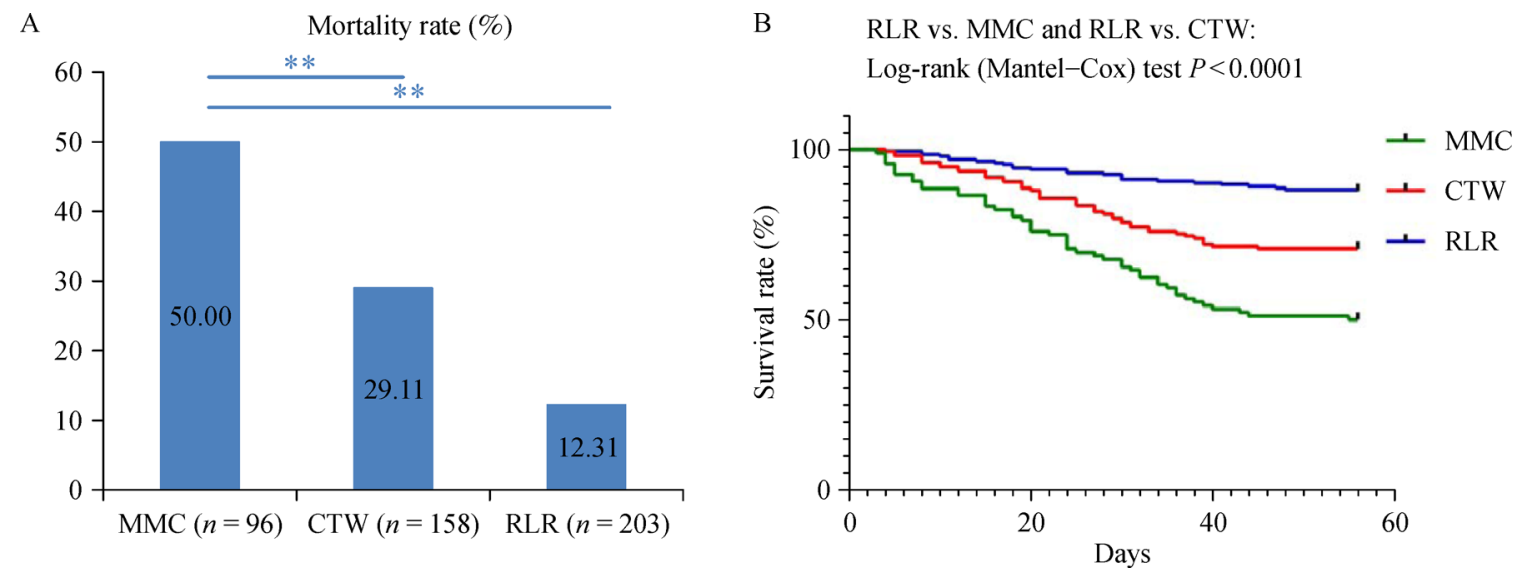

Fig. 2 Mortality rates of patients with HBV-related liver failure in the three groups. (A) The fatality rate in the RLR group was significantly lower than those in the MMC group $(12.31 \%$ vs. $50.00 \%, P=0.000)$ and CTW group $(12.31 \%$ vs. $29.11 \%, P=0.005)$ after 8 weeks of treatment. (B) Survival rates were recorded and analyzed by log-rank (Mantel-Cox) test.

compared with the baseline. However, no significant difference was noted at the end of treatment among these three groups $(P=0.194)$. Moreover, the PTA level in the three groups increased at the end of treatment, and no significant differences were noted among these three groups $(P=0.167)$. The results are shown in Table 3.

\section{Comparison of serum cytokine levels}

After the 8-week treatment period, significant differences in serum cytokine levels were found among the three groups. Furthermore, HGF expression was upregulated in the RLR group, while TGF- $\beta 1$ expression was downregulated when compared with the NC group. The expression levels of SCF, HGF, and VEGF in the RLR group were upregulated but that of TGF- $\beta 1$ was downregulated when compared with the MMC group. Moreover, IFN- $\gamma$ was higher in the RLR group than in the CTW group (Table 4 and Fig. 3).

\section{Safety evaluation}

No abnormal changes in blood routine and renal function were found in the three groups after treatment. Furthermore, no significant adverse reactions related to the two
TCM mixtures used in this study were reported in previous clinical applications. In this work, two patients reported mild adverse reactions, in which one patient in the CTW group had a headache, while another patient in the RLR group had episodes of vomiting. Both symptoms disappeared immediately after proper clinical treatment, indicating that the treatment of "TCM regulating liver regeneration" is safe and effective, with no toxic side effects.

\section{Discussion}

Drug and alcohol abuse has been recognized as the main cause of liver failure in European and American countries. However, the primary cause of liver failure in China is the hepatitis virus (mainly hepatitis B virus), followed by drugs and hepatotoxic substances (such as ethanol, chemical agents, etc.). Acute influencing factors in this study included discontinuation of antiviral agents, ethanol, and exposure to chemical poisons. Acute (subacute)-onchronic liver failure and chronic liver failure have been recorded as the most common clinical symptoms. Patients with HBV-related liver failure are often in severe conditions, with various complications and high mortality.

Table 3 Comparison of biochemical indexes among the three groups before and after treatment $(\bar{x} \pm s)$

\begin{tabular}{llllllllll}
\hline Group & Time & $n$ & PTA $(\%)$ & $n$ & TBIL $(\mu \mathrm{mol} / \mathrm{L})$ & $n$ & ALB $(\mathrm{g} / \mathrm{L})$ & $n$ & ALT $(\mathrm{IU} / \mathrm{L})$ \\
\hline MMC & Before & 93 & $30.62 \pm 12.89$ & 95 & $299.95 \pm 185.45$ & 93 & $31.32 \pm 6.46$ & 95 & $299.34 \pm 393.30$ \\
& After & 54 & $41.73 \pm 21.82$ & 57 & $224.96 \pm 198.61$ & 57 & $32.44 \pm 5.90$ & 57 & $77.98 \pm 186.39$ \\
CTW & Before & 154 & $33.72 \pm 14.20$ & 158 & $291.78 \pm 165.72$ & 158 & $30.45 \pm 5.27$ & 158 & $304.56 \pm 475.07$ \\
& After & 129 & $44.35 \pm 22.80$ & 137 & $233.25 \pm 226.16$ & 137 & $33.11 \pm 5.43$ & 137 & $67.29 \pm 123.70$ \\
\multirow{2}{*}{ RLR } & Before & 203 & $36.56 \pm 15.90$ & 203 & $273.13 \pm 177.27$ & 202 & $32.86 \pm 16.90$ & 203 & $356.51 \pm 532.83$ \\
& After & 181 & $47.71 \pm 22.39$ & 181 & $155.42 \pm 176.17$ & 181 & $35.09 \pm 6.42$ & 181 & $49.87 \pm 73.39$ \\
\hline
\end{tabular}

Normal reference range: PTA, 80\%-120\%; TBIL, 3.4-20.5 $\mu$ mol/L; ALB, 35-55 g/L; ALT, 0-46 IU/L. 
Table 4 Comparison of serum cytokine levels among the different groups

\begin{tabular}{|c|c|c|c|c|}
\hline Cytokine & $\begin{array}{l}\mathrm{NC} \\
(n=10)\end{array}$ & $\begin{array}{l}\text { MMC } \\
(n=10)\end{array}$ & $\begin{array}{l}\text { CTW } \\
(n=10)\end{array}$ & $\begin{array}{l}\text { RLR } \\
(n=5)\end{array}$ \\
\hline$\overline{\mathrm{HGF}}$ & $57.16 \pm 27.81$ & $413.84 \pm 387.89^{\mathbf{\Lambda}}$ & $1162.47 \pm 1264.03^{\mathbf{\Lambda}}$ & $2634.29 \pm 3923.86^{\square \boldsymbol{\Lambda}}$ \\
\hline TGF- $\beta 1$ & $58552.84 \pm 38940.57$ & $40650.32 \pm 23862.58$ & $21952.78 \pm 15136.54^{\Delta \square}$ & $9739.96 \pm 5811.91^{\bullet}$ \\
\hline SCF & $25.48 \pm 6.33$ & $14.93 \pm 6.54^{\boldsymbol{\Perp}}$ & $33.31 \pm 13.65^{\bullet}$ & $28.76 \pm 13.32$ \\
\hline VEGF & $26.14 \pm 19.59$ & $6.26 \pm 8.86^{\boldsymbol{\Lambda}}$ & $10.79 \pm 10.07$ & $14.38 \pm 15.44^{\square}$ \\
\hline IFN- $\gamma$ & $99.88 \pm 125.91$ & $70.04 \pm 62.80$ & $35.91 \pm 58.64$ & $220.51 \pm 295.43^{\bullet}$ \\
\hline TRAIL & $27.24 \pm 18.14$ & $18.32 \pm 5.27$ & $12.11 \pm 16.16^{\Delta \square}$ & $11.03 \pm 9.19$ \\
\hline TNF- $\alpha$ & $6.16 \pm 5.50$ & $15.08 \pm 18.21$ & $29.84 \pm 60.76$ & $30.16 \pm 39.95$ \\
\hline PDGFbb & $587.51 \pm 687.94$ & $445.66 \pm 470.39$ & $212.08 \pm 264.77$ & $92.18 \pm 39.78$ \\
\hline FGF-basic & $12.24 \pm 8.28$ & $7.75 \pm 13.05$ & $6.21 \pm 5.68$ & $9.28 \pm 12.29$ \\
\hline IL-12 & $192.80 \pm 119.08$ & $56.05 \pm 96.54$ & $97.70 \pm 92.18$ & $22.68 \pm 42.88$ \\
\hline IL-18 & $35.04 \pm 20.30$ & $22.72 \pm 11.01$ & $59.11 \pm 35.15$ & $95.51 \pm 118.55$ \\
\hline IL-6 & $18.22 \pm 26.95$ & $12.84 \pm 12.93$ & $106.19 \pm 145.82$ & $44.58 \pm 44.65$ \\
\hline IL-10 & $3.99 \pm 6.27$ & $2.17 \pm 2.44$ & $1.99 \pm 2.34$ & $11.60 \pm 21.38$ \\
\hline IL-13 & $4.89 \pm 6.34$ & $6.10 \pm 4.67$ & $11.82 \pm 16.62$ & $8.89 \pm 13.67$ \\
\hline GCSF & $1.98 \pm 1.01$ & $1.53 \pm 0.88$ & $3.54 \pm 7.35$ & $3.65 \pm 2.74$ \\
\hline GMCSF & $60.65 \pm 37.22$ & $36.17 \pm 27.62$ & $40.52 \pm 30.20$ & $80.02 \pm 113.77$ \\
\hline LIF & $106.45 \pm 55.45$ & $34.70 \pm 44.66$ & $64.08 \pm 59.79$ & $64.35 \pm 24.43$ \\
\hline MIF & $1424.54 \pm 1775.34$ & $797.51 \pm 846.12$ & $1952.77 \pm 2115.81$ & $2343.00 \pm 2045.04$ \\
\hline bNGF & $1.26 \pm 0.43$ & $1.56 \pm 0.45$ & $1.01 \pm 0.73$ & $1.20 \pm 0.55$ \\
\hline
\end{tabular}

Compared with the NC group, ${ }^{\triangle} P<0.05,{ }^{\wedge} P<0.01$; compared with the MMC group, ${ }^{口} P<0.05,{ }^{\bullet} P<0.01$; and compared with the CTW group, $\bullet^{\bullet}<0.01$.

To date, no effective modern medicine therapeutic plan has been developed, and a symptomatic supportive treatment plan is generally used for this serious disease. As recently reported, the combinational therapy of presently approved antiviral drugs with TCM has reduced the mortality of HBV-related liver failure to approximately $30 \%-50 \%$ [8]. Many of these combined treatment plans, which improve the clinical efficacy of liver failure, have resulted in confirmed effectiveness and advancement in reducing mortality and complications and improving life quality by regulating the mechanism of liver damage and regeneration imbalance [9]. The protocol for a multi-center, randomized, and controlled clinical study was adopted to evaluate the efficacy of the combination of antivirals with TCM in treating patients with HBV-related liver failure through the national major and special projects during the Eleventh Five-Year Plan Period. The reported 48-week mortality with combinational therapy was $36.49 \%$, which suggested that the integrated Chinese and Western medicine treatment was significantly effective in reducing the mortality of patients with HBV-related liver failure $[10,11]$. A previous clinical study (ChiCTR-TRC12002961) indicated that the integrated traditional Chinese and Western medicine treatment plan of "TCM regulating liver regeneration" can significantly reduce mortality by up to $16.67 \%$ in patients with HBV-related liver failure; this percentage was significantly lower than that in the control group (16.67\% vs. $51.61 \% ; P<0.05)$. Furthermore, the serum ALB level was significantly higher in the group treated with "TCM regulating liver regeneration" than in the MMC group $(30.72 \pm 2.89$ vs. $28.07 \pm 4.56 ; P<$ $0.05)$ [12]. The results of the present study revealed that the mortality of $12.31 \%$ after the 8 -week treatment was significantly lower in the RLR group than in the MMC group (50\%) and CTW group (29.11\%). Furthermore, the TBIL level was significantly lower in the RLR group $(155.42 \pm 176.17 \mu \mathrm{mol} / \mathrm{L})$ than in the MMC group $(224.96 \pm 198.61 \mu \mathrm{mol} / \mathrm{L})$ and $\mathrm{CTW}$ group $(233.25 \pm 226.16 \mu \mathrm{mol} / \mathrm{L} ; P<0.05)$. The serum ALB level was significantly higher in the RLR group (35.09 \pm $6.42 \mathrm{~g} / \mathrm{L})$ than in the MMC group $(32.44 \pm 5.90 \mathrm{~g} / \mathrm{L})$ and CTW group $(33.11 \pm 5.43 \mathrm{~g} / \mathrm{L} ; P<0.05)$. Moreover, significant differences in serum cytokine levels associated with liver regeneration were detected among the different groups. The expression of SCF, HGF, and VEGF in the RLR group was upregulated, but the expression of TGF $\beta 1$ was downregulated compared with that in the MMC group. The IFN- $\gamma$ level was upregulated in the RLR group compared with that in the CTW group. These results indicated that the treatment of "TCM regulating liver regeneration" can improve liver function, prevent hepatic necrosis, and significantly reduce the complications and mortality of patients.

There is an imbalance between liver injury and liver regeneration in patients with liver failure, and this phenomenon is characterized by excessive injury and 
HGF

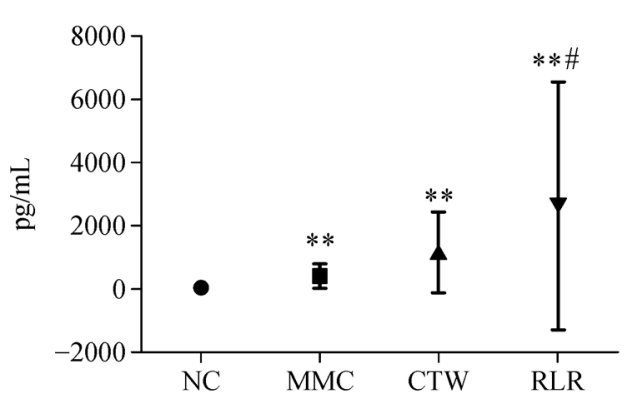

SCF

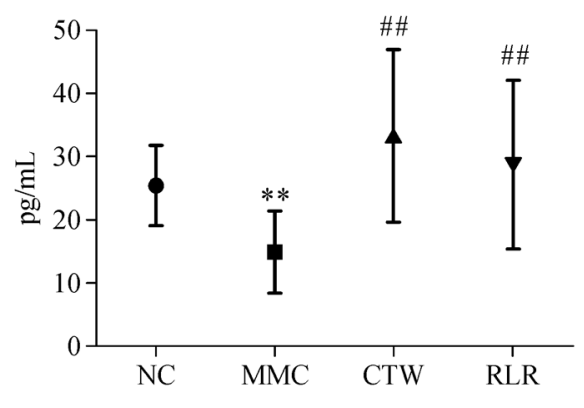

IFN- $\gamma$

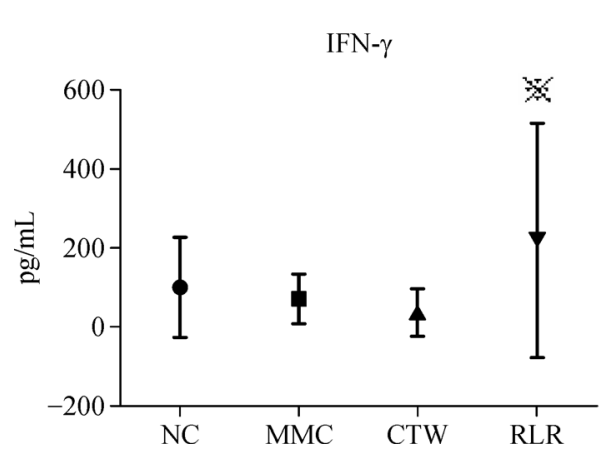

TGF- $\beta 1$
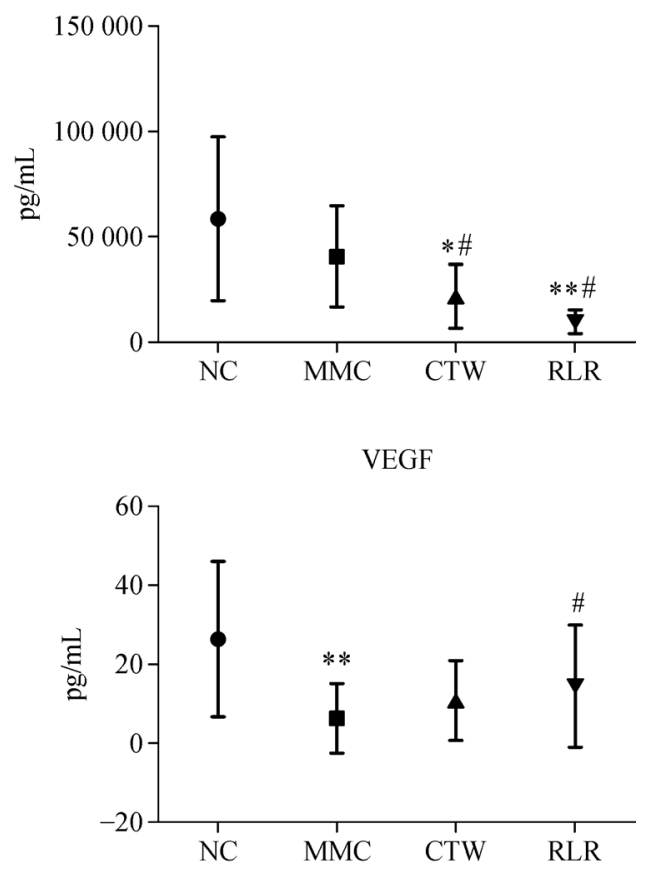

Fig. 3 Serum cytokine levels in patients with CHBLF (pg/mL, mean $\pm \mathrm{SD}$ ). ${ }^{*} P<0.05,{ }^{* *} P<0.01$, vs. the normal control group; ${ }^{\#} P<0.05$, ${ }^{\# \#} P<0.01$, vs. the MMC group; ${ }^{*} P<0.05$, vs. the CTW group.

insufficient regeneration. Thus, the key treatment to rectify this imbalance is to mitigate liver injury, maintain normal liver regeneration, and regulate abnormal liver regeneration $[13,14]$. Liver regeneration is required to restore the damaged parenchymal structure and hepatic function, and it is vital for patient survival. However, when an impaired liver fails to efficiently repair, multi-organ failure with severe outcomes may occur. After systematic studies, the treatment of "TCM regulating liver regeneration" was adopted as a novel therapeutic plan for HBV-related liver failure [13-21]. The treatment of "TCM regulating liver regeneration" can mitigate liver damage, facilitate normal liver regeneration, correct abnormal regeneration, and restore the balance between damage and regeneration by affecting stem cells (liver stem cells, bone marrow stem cells, etc.). Thus, the mortality and life quality of patients can be reduced and improved, respectively.
The previous studies conducted by the investigators revealed that the treatment of "TCM regulating liver regeneration" can improve the conversion rate of bone marrow stem cells into hepatocytes by affecting the liver gene expression profile, which is likely the molecular mechanism for the observed improvement [22-27]. The monosodium glutamate liver regeneration rat model was used to explore the association of liver regeneration with the central nervous system, hypothalamic-pituitary-hepatic axis, and neuro-endocrine-immune network; the treatment of "TCM regulating liver regeneration" delivered a dual regulative effect on liver regeneration and improved hepatic recovery from injury [28-32]. Many clinical studies have suggested that HGF is the most effective cytokine for liver regeneration [33-38]. VEGF, which is the most effective angiogenesis factor for promoting hepatocyte regeneration, also positively affects the hepatic 
regeneration microenvironment [39-41]. SCF can induce the production of progenitor and stem cells, activate them together with other cytokines, and prolong their lives, thereby improving liver regeneration [42-44]. IFN- $\gamma$ is antiviral, immuno-regulatory, and antifibrotic, and it can also alleviate liver injury and promote liver regeneration [43-45]. However, TGF- $\beta$, which inhibits the production of IFN- $\gamma$ and TNF- $\alpha$ in peripheral blood mononuclear cells, negatively impacts liver regeneration by decreasing host immunity and deteriorating liver fibrosis. The results of this study revealed that the treatment of "TCM regulating liver regeneration" improved the expression of HGF, SCF, IFN- $\gamma$, and VEGF and inhibited TGF- $\beta 1$ expression in the treated patients, which led to the reduction in liver injury and apoptosis and promotion of liver regeneration.

Modern pharmacological research has proved that traditional Chinese medicine and its active ingredients in the TCM formula of "TCM regulating liver regeneration" have many functions such as protecting the liver, regulating immunity, and exerting anti-inflammatory and anti-tumor functions. Virgate wormwood herb and its active components have inhibitory effects on influenza virus, hepatitis virus, human immunodeficiency virus, and other viruses $[46,47]$. Indian buead can resist acute and chronic inflammation in different experimental models, and its significant anti-inflammatory effect is widely recognized abroad [48]. Turmeric extract and curcumin can protect the liver through anti-inflammation, antioxidation, and inhibition of fibrosis [49,50]. Glycyrrhizic acid can inhibit hepatocyte apoptosis and liver fibrosis [51]. The total glycosides from common macrocarpium fruit have good anti-inflammatory and immunosuppressive effects [52]. Schisandra chinensis polysaccharide (SCP) significantly reduces liver triglycerides, total cholesterol, alanine aminotransferase, and aspartate aminotransferase [53]. Paeonol has antibacterial, analgesic, and antiinflammatory effects [54]. Rehmannia polysaccharide can improve the hemopoietic function of model mice and enhance the immunity of normal mice $[55,56]$.

The present study was a real-world research, and all clinical and experimental data were obtained and generated by treating and managing a cohort of patients with HBVrelated liver failure. Furthermore, this study summarized our experience, which can be beneficial to others who are interested in improving the outcomes of patients with HBV-related liver failure through combinational therapy with RLR. The real medical environment can clearly reflect the real medical procedures and healthy conditions of patients. Real-world data are collected, stored, and managed primarily through clinical outpatients and inpatient medical records, and privacy is guaranteed by the hospital's quality control department. After obtaining digital and standardized clinical diagnosis and treatment information through statistical analysis, the clinical experience, diagnosis, and treatment rules of TCM can be found from the real clinical environment. Moreover, the multi-dimensionality of the disease can be recognized to evaluate the clinical efficacy. The US Congress posted the "21st Century Cures Act" online, which legalized the replacement of traditional clinical trials with "real-world evidence" to replace traditional clinical trials and expand indications. This real-world research adopts a non-random, distributed treatment strategy, which can treat patients with complicated conditions and multiple diseases and allow clinicians to determine and select treatment plans as they fit the needs of patients depending on their health conditions. This strategy reflects the authenticity of the treatment results. Thus, the real-world research has applicable clinical reference value as supplementary evidence for randomized controlled clinical trials [57]. Given that the sample size of the present study was relatively small, further multi-center and large sample size-based studies are required to verify the reported efficacy. Furthermore, more statistical power should be applied to exclude confounding factors involved in providing higher level evidence for RLR in treating HBV-related liver failure.

\section{Conclusions}

In conclusion, our research suggested that the treatment plan of "TCM regulating liver regeneration" could alleviate jaundice, improve liver function, and significantly reduce the mortality in patients with HBV-related liver failure. The mechanism of action may be that the TCM regulating liver regeneration program can improve the liver regeneration microenvironment (mainly immune and inflammatory microenvironment), regulate immunity and anti-inflammatory function, and manage the imbalance between liver damage and liver regeneration by affecting the expression of cytokines related to liver regeneration. Thus, liver injury is reduced and liver regeneration and repair are promoted.

\section{Acknowledgements}

The present study was financially supported by the National Natural Science Foundation of China (Nos. 81973669, 81703912, and 81603484), the financial support of the Research Project for Practice Development of National TCM Clinical Research Bases (JDZX2015172), and Program of Hanmin Li Famous and Old TCM master' Inheritance Studio.

\section{Compliance with ethics guidelines}

Ling Dai, Xiang Gao, Zhihua Ye, Hanmin Li, Xin Yao, Dingbo Lu, and $\mathrm{Na} \mathrm{Wu}$ declare that they have no conflict of interest. All procedures followed were in accordance with the ethical standards of the responsible committee on human experimentation (institutional 
and national) and with the Helsinki Declaration of 1975, as revised in 2000. The Ethics Committee of Hubei Province Hospital of Traditional Chinese Medicine reviewed and approved the protocol and patient consent form prior to initiation of the study (approval number, 2006001). Informed consent was obtained from all patients who provided blood samples.

\section{References}

1. Ma Z, Wu Y. Current status of liver failure treatment. J Clinic Hepatol (Lin Chuang Gan Dan Bing Za Zhi) 2016; 32: 1668-1672 (in Chinese)

2. Zhao ZG, Han T, Gao YT, Gao YY, Zhang Y, Wu ZP. Causes and clinical outcomes of acute-on-chronic liver failure in patients with hepatitis B virus infection: an analysis of 289 cases. World Chin J Dig (Shi Jie Hua Ren Xiao Hua Za Zhi) 2009; 17(31): 3269-3272 (in Chinese)

3. Organization Committee of 13th Asia-Pacific Congress of Clinical Microbiology and Infection. 13th Asia-Pacific Congress of Clinical Microbiology and Infection Consensus Guidelines for diagnosis and treatment of liver failure. Hepatobiliary Pancreat Dis Int 2013; 12(4): 346-354

4. Ning Q, Zhu L, Yan WM. Prewarning of severe acute aggravation of chronic hepatitis B. Chin J Hepatol (Zhonghua Gan Zang Bing Za Zhi) 2010; 18(11): 805-807 (in Chinese)

5. Tan XM, Ning Q. The progress in molecular mechanism of severe hepatitis. Int J Epidemiol Infect Dis (Guo Ji Liu Xing Bing Xue Chuan Ran Bing Xue Za Zhi) 2004; 31: 150-157 (in Chinese)

6. Li HM. Basic and clinical research of traditional Chinese medicine regulating and controlling liver regeneration. Chin Arch Tradit Chin Med (Zhonghua Zhong Yi Yao Xue Kan) 2017; 35: 1927-1931 (in Chinese)

7. Liver Failure and Artificial Liver Group, Chinese Society of Infectious Diseases, Chinese Medical Association. Diagnostic and treatment guidelines for liver failure (2012 version). Chin J Hepatol (Zhonghua Gan Zang Bing Za Zhi) 2013; 21(3): 177-183 (in Chinese)

8. Zhou SN, Zhang N, Wang LF, Li J. Clinical research of hepatitis Brelated liver failure by TCM and WM combination therapy. Chin Hepatol (Gan Zang) 2012; 17: 36-38 (in Chinese)

9. Li HM. Progress and prospect of regulation of liver regeneration by traditional Chinese medicine. World Chin J Dig (Shi Jie Hua Ren Xiao Hua Za Zhi) 2017; 25(15): 1338 (in Chinese)

10. Wang LF, Li J, Li FY. Analysis the curative efficacy of combination of Chinese and Western medicine treatment the hepatitis B virus associated hepatic failure. Chin J Integr Tradit West Med Liver Dis (Zhong Xi Yi Jie He Gan Bing Za Zhi) 2018; 28: 70-74 (in Chinese)

11. Gong M, Zhou C, Zhang N. Clinical effect of integrated traditional Chinese and Western medicine therapy in patients with hepatitis B virus-related acute-on-chronic liver failure complicated by hepatic encephalopathy. J Clinic Hepatol (Lin Chuang Gan Dan Bing Za Zhi) 2018; 34: 795-800 (in Chinese)

12. Li HM, Ye ZH, Zhang J, Gao X, Chen YM, Yao X, Gu JX, Zhan L, Ji Y, Xu JL, Zeng YH, Yang F, Xiao L, Sheng GG, Xin W, Long Q, Zhu QJ, Shi ZH, Ruan LG, Yang JY, Li CC, Wu HB, Chen SD, Luo
XL. Clinical trial with traditional Chinese medicine intervention "tonifying the kidney to promote liver regeneration and repair by affecting stem cells and their microenvironment" for chronic hepatitis B-associated liver failure. World J Gastroenterol 2014; 20(48): 18458-18465

13. Li HM. Discussion on the treatment principle of "tonifying the kidney to regulate stem cells transforming the liver". Chin Arch Tradit Chin Med (Zhonghua Zhong Yi Yao Xue Kan) 2012; 30 : 937-940 (in Chinese)

14. Li HM. Regulation of liver regeneration: research progress and prospect. World Chin J Dig (Shi Jie Hua Ren Xiao Hua Za Zhi) 2015; 23(21): 3337-3343 (in Chinese)

15. Li HM. The unbalance of EMT/MET and "marrow cells cannot differentiate into liver cells". Chin J Integr Tradit West Med Liver Dis (Zhong Xi Yi Jie He Gan Bing Za Zhi) 2012; 21: 385-389 (in Chinese)

16. Li HM. Evolution rule of jaundice syndromes in chronic sever hepatitis. Chin J Integr Tradit West Med Liver Dis (Zhong Xi Yi Jie He Gan Bing Za Zhi) 2009; 19: 148-150 (in Chinese)

17. Li HM. Comprehensive and systematic in-depth study on traditional Chinese medicine for regulating liver regeneration. Chin J Integr Tradit West Med Liver Dis (Zhong Xi Yi Jie He Gan Bing Za Zhi) 2007; 17: 129-132 (in Chinese)

18. Li HM, Gao X, Yan XS, Ming AP, Peng YQ. Study on molecular mechanism of Zuoguiwan in promoting bone marrow cells to form hepatic cells. J Tradit Chin Med (Zhong Yi Za Zhi) 2006; 47: 778780 (in Chinese)

19. Song HL, Li HM, Lin LS, Gao X, Zhao BB, Zhang J, Wu Y, Yan XS, Xiao L. Effects of Diwuyanggan capsule on liver regeneration of rat with deficiency of kidney essence and liver blood. Chin J Integr Tradit West Med Liver Dis (Zhong Xi Yi Jie He Gan Bing Za Zhi) 2013; 23: 90-92 (in Chinese)

20. Li HM. Basic and clinical research of "tonifying the kidney to regulate stem cells transforming the liver" for liver diseases. World Sci Technol/Modernization Tradit Chin Med Mater Med (Shi Jie Ke Xue Ji Shu-Zhong Yi Yao Xian Dai Hua) 2013; 15: 1425-1428 (in Chinese)

21. Gao X, Ye ZH, Dai L, Li HM. Molecular mechanism research of Zuogui Pill about "tonifying kidney to promote liver regeneration and repair by effecting stem cells and their microenvironment" by using the network pharmacology method. Chin J Integr Tradit West Med Liver Dis (Zhong Xi Yi Jie He Gan Bing Za Zhi) 2018; 28: $96-$ 99 (in Chinese)

22. Li HM, Gao X, Yan XS, Ming AP, Peng YQ, Li JJ. Promotion effect of Zuoguiwan on bone marrow cells' forming into liver cells in mice. World Chin J Dig (Shi Jie Hua Ren Xiao Hua Za Zhi) 2005; 13: 2818-2822 (in Chinese)

23. Li HM, Xiang G, Yan XS. Studies of Zuogui Wan medicated serum pharmacology based on the co-culture system of bone marrow stem cells and hepatocytes. J Clin Rehabil Tissue Eng Res (Zhongguo Zu Zhi Gong Cheng Yan Jiu Yu Lin Chuang Kang Fu) 2010; 14: $3527-$ 3532 (in Chinese)

24. Li HM, Yan XS, Luo JJ, Li JJ, Gao X, Ming AP, Peng YQ. Effect of Zuogui Wan drug serum on the differentiation of bone marrow mesenchymal stem cells into hepatocytes. J Clin Rehabil Tissue Eng Res (Zhongguo Zu Zhi Gong Cheng Yan Jiu Yu Lin Chuang Kang Fu) 2007; 11: 5465-5468 (in Chinese) 
25. Li HM, Yan XS, Ming AP, Peng YQ, Luo JJ, Lan SB, Gao X. The differentiation of marrow stromal cells into hepatocyte by conditioned medium of liver cells in vitro. Chin J Integr Tradit West Med Liver Dis (Zhong Xi Yi Jie He Gan Bing Za Zhi) 2005; 15: 28-30, 67 (in Chinese)

26. Li HM, Gui WJ, Li JJ, Gao X, Yan XS, Cheng Y. Effects of Zuoguiwan on the liver regeneration related gene signaling pathway in female mice with male mice bone marrow transplant. J Clin Rehabil Tissue Eng Res (Zhongguo Zu Zhi Gong Cheng Yan Jiu Yu Lin Chuang Kang Fu) 2008; 12: 6069-6073 (in Chinese)

27. Li HM, Gao X, Yan XS, Ming AP, Peng YQ. Analysis of gene expression profile of bone marrow cells forming liver cells. Chin J Integr Tradit West Med Liver Dis (Zhong Xi Yi Jie He Gan Bing Za Zhi) 2006; 16: 212-214, 217 (in Chinese)

28. Li HM, Gao X, Zhou MS. Effects of Zuogui Pill on gene expression profile of regenerate hepatic tissue in MSG-liver regeneration-rat. China J Tradit Chin Med Pharm (Zhonghua Zhong Yi Yao Za Zhi) 2006; 21: 104-106 (in Chinese)

29. Li HM, Gao X, Zhou MS. Zuogui Pill regulating gene expression profile in liver of MSG-induced liver regeneration in rats. Chin J Basic Med Tradit Chin Med (Zhongguo Zhong Yi Ji Chu Yi Xue Za Zhi) 2005; 11: 595-598 (in Chinese)

30. Li HM, Yang ML, Mei JJ, Zhang LT, Qiu XF. The effects of Zuogui Pill on expression of TGF- $\alpha, \beta$ and it's receptor in ARN and regenerative liver of the MSG-regeneration-rat. Chin J Hepatol (Zhonghua Gan Zang Bing Za Zhi) 2004; 12(5): 307-308 (in Chinese)

31. Li HM, Gao X, Yang ML, Mei JJ, Zhang LT, Qiu XF. Effects of Zuogui Wan on neurocyte apoptosis and down-regulation of TGF$\beta 1$ expression in nuclei of arcuate hypothalamus of monosodium glutamate-liver regeneration rats. World J Gastroenterol 2004; 10(19): 2823-2826

32. Li HM, Yang ML, Mei JJ, Zhang LT, Qiu XF. Apoptosis of hypothalamic neural cell and genetic expression of TGF- $\beta 1$ in MSG-liver regeneration-rats. Chin J Appl Physiol (Zhongguo Ying Yong Sheng Li Xue Za Zhi) 2003; 19: 46-47, 93 (in Chinese)

33. Xu CL, Hao YH, Lu YP, Tang ZS, Yang XC, Wu J, Zheng X, Wang BJ, Liu J, Yang DL. Upregulation of Toll-like receptor 4 on T cells in PBMCs is associated with disease aggravation of HBV-related acute-on-chronic liver failure. J Huazhong Univ Sci Technolog Med Sci 2015; 35(6): 910-915

34. Zhao ZH, Fan YC, Zhao Q, Dou CY, Ji XF, Zhao J, Gao S, Li XY, Wang K. Promoter methylation status and expression of PPAR- $\gamma$ gene are associated with prognosis of acute-on-chronic hepatitis $\mathrm{B}$ liver failure. Clin Epigenetics 2015; 7(1): 115

35. Fujiwara K, Yasui S, Yokosuka O. Corticosteroid and nucleoside analogue for hepatitis B virus-related acute liver failure. World $\mathrm{J}$ Gastroenterol 2015; 21(36): 10485-10486

36. Cao Z, Li F, Xiang X, Liu K, Liu Y, Tang W, Lin L, Guo Q, Bao S, Xie Q, Wang H. Circulating cell death biomarker: good candidates of prognostic indicator for patients with hepatitis B virus related acute-on-chronic liver failure. Sci Rep 2015; 5(1): 14240

37. Wang J, Yu Y, Yang Y, Wu SS, Zhu HH, Liu YN, Liu WX, Hu Y, Wu W, Xia CX, Chen Z. Expression of serum sCD163 in patients with liver diseases and inflammatory disorders. Int J Clin Exp Pathol 2015; 8(7): 8419-8425

38. Porowski D, Wirkowska A, Hryniewiecka E, Wyzgał J, Pacholczyk
M, Pączek L. Liver failure impairs the intrahepatic elimination of interleukin-6, tumor necrosis factor- $\alpha$, hepatocyte growth factor, and transforming growth factor- $\beta$. BioMed Res Int 2015; 2015: 934065

39. Zeng W, Xiao J, Zheng G, Xing F, Tipoe GL, Wang X, He C, Chen ZY, Liu Y. Antioxidant treatment enhances human mesenchymal stem cell anti-stress ability and therapeutic efficacy in an acute liver failure model. Sci Rep 2015; 5(1): 11100

40. Manzini BM, da Silva Santos Duarte A, Sankaramanivel S, Ramos AL, Latuf-Filho P, Escanhoela C, Kharmandayan P, Olalla Saad ST, Boin I, Malheiros Luzo AC. Useful properties of undifferentiated mesenchymal stromal cells and adipose tissue as the source in liverregenerative therapy studied in an animal model of severe acute fulminant hepatitis. Cytotherapy 2015; 17(8): 1052-1065

41. Ma HC, Shi XL, Ren HZ, Yuan XW, Ding YT. Targeted migration of mesenchymal stem cells modified with CXCR4 to acute failing liver improves liver regeneration. World J Gastroenterol 2014; 20(40): 14884-14894

42. Chiang $\mathrm{CH}, \mathrm{Wu} \mathrm{WW}$, Li HY, Chien Y, Sun CC, Peng CH, Lin AT, Huang CS, Lai YH, Chiou SH, Hung SI, Chang YL, Lan YT, Liu DM, Chien CS, Huo TI, Lee SD, Wang CY. Enhanced antioxidant capacity of dental pulp-derived iPSC-differentiated hepatocytes and liver regeneration by injectable HGF-releasing hydrogel in fulminant hepatic failure. Cell Transplant 2015; 24(3): 541-559

43. Cienfuegos JA, Rotellar F, Baixauli J, Martínez-Regueira F, Pardo F, Hernández-Lizoáin JL. Liver regeneration - the best kept secret. A model of tissue injury response. Rev Esp Enferm Dig 2014; 106(3): 171-194

44. Xiao JQ, Shi XL, Tan JJ, Zhang L, Xu Q, Ding YT. A novel treatment regimen for acute liver failure based on a combination of mesenchymal stem cells transplantation and IL-lRa-loaded chitosan nanoparticles. Chin J Hepatol (Zhonghua Gan Zang Bing Za Zhi) 2013; 21(4): 308-314 (in Chinese)

45. Xiao JQ, Shi XL, Ma HC, Tan JJ, zhang L, Xu Q, Ding YT. Administration of IL-1Ra chitosan nanoparticles enhances the therapeutic efficacy of mesenchymal stem cell transplantation in acute liver failure. Arch Med Res 2013; 44(5): 370-379

46. Yoon M, Kim MY. The anti-angiogenic herbal composition Ob-X from Morus alba, Melissa officinalis, and Artemisia capillaris regulates obesity in genetically obese ob/ob mice. Pharm Biol 2011; 49(6): 614-619

47. Zhang T, Chen D. Anticomplementary principles of a Chinese multiherb remedy for the treatment and prevention of SARS. J Ethnopharmacol 2008; 117(2): 351-361

48. Ríos JL. Chemical constituents and pharmacological properties of Poria cocos. Planta Med 2011; 77(7): 681-691

49. Lee HY, Kim SW, Lee GH, Choi MK, Chung HW, Lee YC, Kim HR, Kwon HJ, Chae HJ. Curcumin and Curcuma longa L. extract ameliorate lipid accumulation through the regulation of the endoplasmic reticulum redox and ER stress. Sci Rep 2017; 7(1): 6513

50. Uchio R, Higashi Y, Kohama Y, Kawasaki K, Hirao T, Muroyama $\mathrm{K}$, Murosaki S. A hot water extract of turmeric (Curcuma longa) suppresses acute ethanol-induced liver injury in mice by inhibiting hepatic oxidative stress and inflammatory cytokine production. $\mathrm{J}$ Nutr Sci 2017; 6: e3

51. Liang B, Guo XL, Jin J, Ma YC, Feng ZQ. Glycyrrhizic acid inhibits apoptosis and fibrosis in carbon-tetrachloride-induced rat liver 
injury. World J Gastroenterol 2015; 21(17): 5271-5280

52. Isaza $\mathrm{JH}$, Ito $\mathrm{H}$, Yoshida $\mathrm{T}$. A flavonol glycoside-lignan ester and accompanying acylated glucosides from Monochaetum multiflorum. Phytochemistry 2001; 58(2): 321-327

53. Wang CM, Yuan RS, Zhuang WY, Sun JH, Wu JY, Li H, Chen JG. Schisandra polysaccharide inhibits hepatic lipid accumulation by downregulating expression of SREBPs in NAFLD mice. Lipids Health Dis 2016; 15(1): 195

54. Liao WY, Tsai TH, Ho TY, Lin YW, Cheng CY, Hsieh CL. Neuroprotective effect of paeonol mediates anti-inflammation via suppressing Toll-like receptor 2 and Toll-like receptor 4 signaling pathways in cerebral ischemia-reperfusion injured rats. Evid Based Complement Alternat Med 2016; 2016: 3704647
55. Liu PJ, Miao MS, Gao JL. Effect of gross polysaccharide of Radix Rehmanniae Preparata on levels of whole blood cells and serum macrophage colony stimulating factor in mice with both qi and blood deficiency. J Clin Rehabil Tissue Eng Res 2008; 12: 75437546

56. Li FS, Xu HG, Li MY, Liu H. Stndy on immunological properties of polysaccharide extracted from rehmanniae preparata. Chin J Publ Health 2008; 24: 1109-1110

57. Sherman RE, Anderson SA, Dal Pan GJ, Gray GW, Gross T, Hunter NL, LaVange L, Marinac-Dabic D, Marks PW, Robb MA, Shuren J, Temple R, Woodcock J, Yue LQ, Califf RM. Real-world evidence - what is it and what can it tell us? N Engl J Med 2016; 375(23): 2293-2297 